\title{
Scaling of Raman amplification in realistic slow-light photonic crystal waveguides
}

\author{
Isabella H. Rey, ${ }^{1, *}$ Yannick Lefevre, ${ }^{2}$ Sebastian A. Schulz, ${ }^{1}$ Nathalie Vermeulen, ${ }^{2}$ and Thomas F. Krauss ${ }^{1}$ \\ ${ }^{1}$ School of Physics and Astronomy, University of St Andrews, St Andrews, Fife KY16 9SS, United Kingdom \\ ${ }^{2}$ Brussels Photonics Team (B-PHOT), Department of Applied Physics and Photonics, Vrije Universiteit Brussel, B-1050 Brussels, Belgium
}

(Received 13 April 2011; revised manuscript received 6 June 2011; published 20 July 2011)

\begin{abstract}
The prospect for low pump-power Raman amplification in silicon waveguides has recently been boosted by theoretical studies discussing the enhancement of nonlinear phenomena in slow-light structures. In principle, the slowing down of either the pump or the signal beam is equivalent in terms of Raman gain, but in the presence of losses, we show that they play different roles in determining the net signal gain. We also investigate the impact of the mode profile in realistic slow-light waveguides on the total gain, an effect that is usually neglected in the context of stimulated Raman scattering. By taking representative losses and mode shapes into account, we provide a realistic estimation of the achievable performance of slow-light photonic crystal waveguides.
\end{abstract}

DOI: 10.1103/PhysRevB.84.035306

PACS number(s): 42.65.Dr, 42.70.Qs, 42.82.Et

\section{INTRODUCTION}

Slow light has recently generated a lot of interest in the field of nonlinear photonics because it facilitates increased light-matter interaction and, thus, enhanced nonlinear effects, which has the prospect for reducing the device footprint and the required pump power. ${ }^{1}$ This enhancement has already been demonstrated for a number of nonlinear phenomena, including four-wave mixing (FWM), ${ }^{2}$ self-phase modulation, ${ }^{3}$ and third harmonic generation. ${ }^{4}$

Another nonlinear effect for which slow light holds great promise is stimulated Raman scattering (SRS), especially in silicon due to its high Raman scattering cross section. The inverse dependence of the Raman gain on the group velocities of both the pump and the Stokes signals was first proposed by McMillan et al., ${ }^{5}$ predicting an increase of the Raman gain by more than a factor of $10^{4}$ with respect to conventional "fast-light" structures. This has been followed by an experimental demonstration of slow-light-enhanced spontaneous Raman scattering ${ }^{6}$ and, more recently, stimulated Raman scattering ${ }^{7}$ in slow-light waveguides.

One of the key questions when considering slow-lightenhanced Raman gain is whether it is better to slow down the pump or the Stokes signal; it is reasonable to assume, from first principles, that slowing down either one has the same impact on the Raman gain. ${ }^{5}$ In the presence of losses, however, this question becomes less obvious and has not yet been satisfactorily addressed. Following a first investigation carried out by Krause et al., ${ }^{8}$ we present here a simple and intuitive description showing that slowing down the pump or the Stokes signal yields intrinsically different results if realistic losses are present.

We then proceed by analyzing the influence of the modeprofile variation with slowdown factor on the signal gain, an effect that has already been proven to be important for the accurate determination of linear losses ${ }^{9}$ and FWM efficiency ${ }^{2}$ in photonic crystal (PhC) waveguides, but which has so far been neglected for the case of Raman gain. ${ }^{8}$ We present an estimate of the gain achievable with realistic slow-light $\mathrm{PhC}$ waveguides, showing that low pump-power operation requires both slowing down and careful attention to propagation loss and mode-profile effects. In short, the key question motivating this paper is how to maximize the Raman gain for a given input power by slowing down either the pump or the Stokes signal.

\section{SIMPLIFIED MODEL: THE DIFFERENCE BETWEEN $S_{s}$ AND $S_{p}$}

We consider the case in which a continuous-wave $(\mathrm{CW})$ signal at frequency $\omega_{s}$ copropagates in a silicon slow-light waveguide with a CW pump at frequency $\omega_{p}$, where $\omega_{p}-$ $\omega_{s}$ is equal to the Raman shift of silicon $\Delta \omega_{R}=2 \pi \times$ 15.6 THz. The slowing down of each of the two beams inside the waveguide is quantified by the slowdown factor $S$, which is defined as the ratio between the group index and the material refractive index $S=n_{g} / n_{\mathrm{Si}}$. Taking the strong pump assumption approximation into account, ${ }^{10}$ the evolution of the pump and signal intensities $I_{p}(z)$ and $I_{s}(z)$, respectively, is then governed by the following equations ${ }^{8,11}$ :

$$
\begin{aligned}
\frac{\partial I_{p}}{\partial z}= & -\alpha_{p}\left(S_{p}\right) I_{p}-\beta_{\mathrm{TPA}} S_{p}^{2} I_{p}^{2}-\frac{\sigma_{p} \beta_{\mathrm{TPA}} \tau_{c}}{2 \hbar \omega_{p}} S_{p}^{3} I_{p}^{3}, \\
\frac{\partial I_{s}}{\partial z}= & -\alpha_{s}\left(S_{s}\right) I_{s}+\left(g_{R}-2 \beta_{\mathrm{TPA}}\right) S_{p} S_{s} I_{p} I_{s} \\
& -\frac{\sigma_{s} \beta_{\mathrm{TPA}} \tau_{c}}{2 \hbar \omega_{p}} S_{p}^{2} S_{s} I_{p}^{2} I_{s} .
\end{aligned}
$$

In Eqs. (1) and (2), the first term on the right-hand side accounts for linear loss, the second term describes two-photon absorption (TPA) through the TPA coefficient $\beta_{\text {TPA }}$, and, in the signal equation, SRS through the Raman gain $g_{R}$. The third term accounts for TPA-induced free-carrier absorption (FCA), with $\sigma_{p, s}$ the FCA coefficients for pump and signal, and $\tau_{c}$ the free-carrier lifetime. The key question is to determine how the respective parameters scale with the slowdown factor $S$ : all nonlinearities are scaled by the slowdown factors $S_{p}$ and $S_{s}$ of pump and signal, respectively, as shown in Eqs. (1) and (2). ${ }^{8}$

If the linear loss is dominated by out-of plane scattering, the first term on the right-hand side of Eqs. (1) and (2) scales, to first approximation, linearly with $S,{ }^{12,13}$ i.e., $\alpha_{i}\left(S_{i}\right)=\kappa_{i} S_{i}$, with $\kappa_{i}$ the (constant) loss per unit slowdown factor. Such linear scaling has already been observed up to group indices of $n_{g}=40$ and is expected to be achievable up to $n_{g}=60-80$ with novel designs. ${ }^{14}$ Equation (2) can therefore be rewritten in terms of a Stokes gain function $G_{s}$ as

$$
\frac{\partial I_{s}}{\partial z}=G_{s}\left(S_{p} I_{p}\right) S_{s} I_{s},
$$



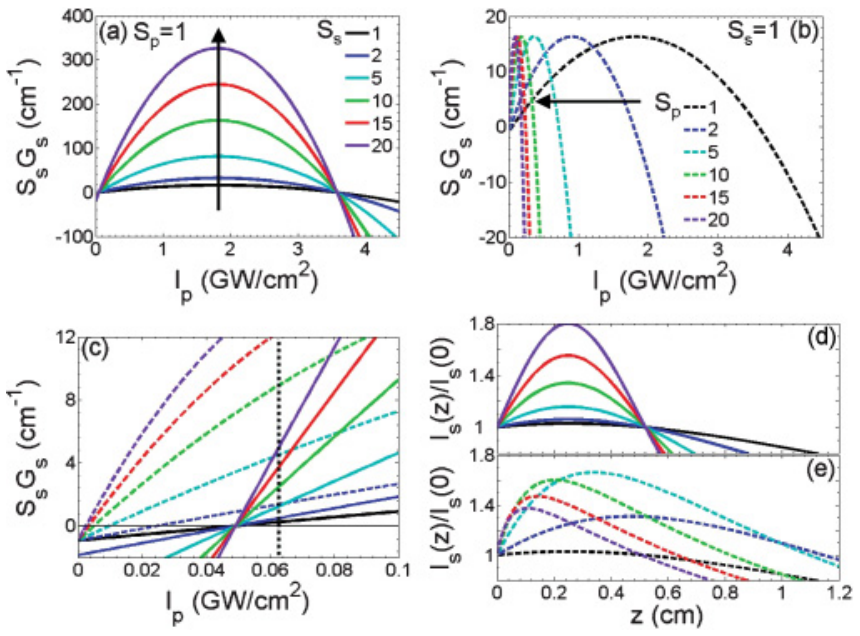

FIG. 1. (Color online) (a), (b) Signal gain function $S_{s} G_{s}\left(I_{p}\right)$ as computed using the parameters given in Table I for different values of (a) $S_{s}$ and (b) $S_{p}$. (c) Zoom of (a) and (b) for $I_{p}$ up to $0.1 \mathrm{GW} / \mathrm{cm}^{2}$; the vertical dotted line indicates the input pump intensity $I_{p}(0)$ used for the plots in (d) and (e); the chosen value of $I_{p}(0)$ corresponds to a $200-\mathrm{mW}$ input pump power into a waveguide with effective area $0.32 \mu \mathrm{m}^{2}$. (d), (e) Evolution of the signal intensity along the waveguide ( $z$ direction) for the same values of (d) $S_{s}$ and (e) $S_{p}$ as in the previous graphs.

where

$$
G_{s}\left(S_{p} I_{p}\right)=-\kappa_{s}+\left(g_{R}-2 \beta_{\mathrm{TPA}}\right) S_{p} I_{p}-\frac{\sigma_{s} \beta_{\mathrm{TPA}} \tau_{c}}{2 \hbar \omega_{p}} S_{p}^{2} I_{p}^{2}
$$

is a quadratic function of the product $S_{p} I_{p}$. Figures 1(a) and 1(b) show how the product $G_{s} S_{s}$ varies as a function of $I_{p}$, when computed using the material parameters given in Table I.

From Eqs. (3) and (4), the difference between $S_{s}$ and $S_{p}$ is evident: whether there is gain or loss depends on the sign of $G_{s}$, which is solely a function of the pump intensity $S_{p} I_{p}$ and thus not related to $S_{s}$. Since $S_{s}$ is an overall multiplying factor, it only enhances the already experienced gain or loss, which shows as a scaling along the $y$ axis in Fig. 1(a). This also means that $S_{s}$ has no influence on the threshold pump intensity $I_{p, t h}$, i.e., the (lowest) value of $I_{p}$ for which $G_{s}=0$ [Fig. 1(c), solid curves]:

$$
\begin{aligned}
I_{p, t h}= & \frac{g_{R}-2 \beta_{\mathrm{TPA}}}{S_{p}} \frac{\hbar \omega_{p}}{\sigma_{s} \beta_{\mathrm{TPA}} \tau_{c}} \\
& \times\left[1-\sqrt{1-\frac{2 \kappa_{s}}{\left(g_{R}-2 \beta_{\mathrm{TPA}}\right)^{2}} \frac{\sigma_{s} \beta_{\mathrm{TPA}} \tau_{c}}{\hbar \omega_{p}}}\right] .
\end{aligned}
$$

TABLE I. Values of the parameters used in the calculations.

\begin{tabular}{lcc}
\hline \hline Parmeter name & Symbol & Value \\
\hline Loss per unit $S$ & $\kappa_{s, p}$ & $4 \mathrm{~dB} / \mathrm{cm} / \mathrm{S}=1.15 \mathrm{~dB} / \mathrm{cm} / \mathrm{n}_{\mathrm{g}}$ (Ref. 14) \\
Stokes wavelength & $\lambda_{s}$ & $1550 \mathrm{~nm}$ \\
Raman gain & $g_{R}$ & $20 \mathrm{~cm} / \mathrm{GW}$ \\
TPA coefficient & $\beta_{\mathrm{TPA}}$ & $0.5 \mathrm{~cm} / \mathrm{GW}$ \\
Carrier lifetime & $\tau_{c}$ & $200 \mathrm{ps}($ Ref. 15) \\
FCA coefficient & $\sigma_{s, p}$ & $1.45 \times 10^{17}\left(\lambda_{s, p} / 1550 \mathrm{~nm}\right)^{2} \mathrm{~cm}^{2}$ \\
\hline \hline
\end{tabular}

On the other hand, the slowdown factor $S_{p}$ of the pump corresponds to a scaling of the Stokes gain function $G_{s}$ on the $x$ axis [Fig. 1(b)] and therefore determines for which pump intensities $G_{s}$ is positive; by slowing down the pump, the pump threshold $I_{p, t h}$ given by Eq. (5) is scaled down by a factor $S_{p}$ [Fig. 1(c), dashed curves]. Yet, the effect of $S_{p}$ on the signal evolution along the waveguide is more complicated because slowing down the pump also causes a faster decay of the pump intensity due to linear loss TPA and FCA [Eq. (1)]. This results in the existence of an optimal pump slowdown factor $S_{p}$ for a given input pump intensity, after which a further increase of $S_{p}$ gives no benefit in terms of maximum achievable net gain ${ }^{16}$ [i.e., the curve of Fig. 1(e) has lower peak for high $S_{p}$ ], as also indicated by Krause et al. ${ }^{8}$ If instead $S_{S}$ is increased, the gain function enhancement is not accompanied by a faster decay of the pump, and therefore, in principle, the maximum gain [the curve peak in Fig. 1(d)] always increases with $S_{s}$.

It should be noted that the shape of the signal gain function $G_{s}\left(I_{p}\right)$ is greatly sensitive to variations in the material parameters. The pump threshold $I_{p, t h}$, for instance, depends heavily on the signal linear loss per unit slowdown factor $\kappa_{s}=\alpha_{s} / S_{s}$ and on the Raman gain coefficient $g_{R}$. The loss is clearly dependent on technology, but typical values between $1-2 \mathrm{~dB} / \mathrm{cm} / \mathrm{n}_{\mathrm{g}}$ have been reported, ${ }^{14}$ while reported values for the Raman gain vary over a wide range $(4.3-76 \mathrm{~cm} / \mathrm{GW}) .{ }^{17-19}$ Note that we have chosen a realistically achievable value for $\kappa$ (Ref. 14) and a conservative value for $g_{R}$.

Finally, it is worth pointing out that, for the pump intensities and low carrier lifetime considered here [we estimate $\tau_{c}=200 \mathrm{ps}$ as a reasonable upper-bound value for $\mathrm{PhCs}$ (Ref. 15)], FCA due to pump-generated carriers, although not completely negligible, does not represent the main obstacle for achieving signal gain under $\mathrm{CW}$ pump operation, as it was instead believed in previous works. ${ }^{7}$ We rather identify the main limitation in the signal linear propagation loss $\alpha_{s}\left[\kappa_{s}\right.$ is the $y$ intercept in Figs. 1(a)-1(c)], which needs to be overcome before threshold can be reached and the structure can exhibit net gain; thus, the need for low-loss slow-light waveguides is strongly emphasized. Of course, if the structure design allows for slowing down both pump and signal at the same time, one would greatly benefit from the advantages of both configurations, ${ }^{8}$ although it is challenging to realize designs with practically achievable loss values and tolerances.

\section{SRS IN REALISTIC PHC WAVEGUIDES}

The model described in the preceding section, while giving a useful and clear insight into the main difference between slowing down the Raman pump and the Stokes signal, does not capture the full picture of dealing with real-life slow-light $\mathrm{PhC}$ waveguides. In such waveguides, the propagation loss $\alpha$ does not necessarily vary linearly with $S$ and the mode profile may depend on the slowdown factor. ${ }^{9}$ The mode-profile variation causes changes of the overlap between pump and signal modes, which influences the effective value of the Raman gain $g_{R} \cdot{ }^{5}$ Moreover, from an experimental point of view, we are mostly interested in the achievable performance in terms of input pump power $P_{p 0}=I_{p 0} A_{\text {eff, } p}$, which ideally 
should be as low as possible in order to enable a useful application of Raman gain in integrated photonic devices. Therefore, the most important parameter that we need to add to the analysis is the effective mode area $A_{\text {eff }}$, especially as we have already shown its importance experimentally for the case of FWM. ${ }^{2}$

We therefore rewrite Eqs. (1) and (2) in terms of the CW pump and signal powers $P_{p}$ and $P_{s}$, respectively, and we include in each nonlinear term a scaling coefficient $c_{k}$ to describe the mode overlap ${ }^{5,20}$ :

$$
\begin{aligned}
\frac{\partial P_{p}}{\partial z}= & -\alpha_{p}\left(S_{p}\right) P_{p}-\beta_{\mathrm{TPA}} c_{\mathrm{TPA}, p p} \frac{S_{p}^{2}}{A_{\mathrm{eff}, p}} P_{p}^{2} \\
& -\frac{\sigma_{p} \beta_{\mathrm{TPA}} \tau_{c}}{2 \hbar \omega_{p}} c_{\mathrm{FCA}, p p} \frac{S_{p}^{3}}{A_{\mathrm{eff}, p}^{2}} P_{p}^{3}, \\
\frac{\partial P_{s}}{\partial z}= & {\left[-\alpha_{s}\left(S_{s}\right)+\left(g_{R} c_{R}-2 \beta_{\mathrm{TPA}} c_{\mathrm{TPA}, s p}\right) \frac{S_{p} S_{s}}{A_{\mathrm{eff}, p}} P_{p}\right.} \\
& \left.-\frac{\sigma_{s} \beta_{\mathrm{TPA}} \tau_{c}}{2 \hbar \omega_{p}} c_{\mathrm{FCA}, s p} \frac{S_{p}^{2} S_{s}}{A_{\mathrm{eff}, p}^{2}} P_{p}^{2}\right] P_{s},
\end{aligned}
$$

with

$$
\begin{aligned}
c_{R} & =\frac{A_{\mathrm{eff}, p}}{A_{R}}, \\
c_{\mathrm{TPA}, i p} & =\frac{A_{\mathrm{eff}, p}}{A_{\mathrm{TPA}, i p}}, \\
c_{\mathrm{FCA}, i p} & =\frac{A_{\mathrm{eff}, p}^{2}}{A_{\mathrm{FCA}, i p}^{2}},
\end{aligned}
$$

where $A_{k}$ are the nonlinear interaction effective areas. The Raman effective area $A_{R}$, for instance, is determined from the mode profiles $\mathbf{E}_{i}(\mathbf{r})$ as

$$
A_{R}=\frac{\left[\int_{V_{\mathrm{tot}}} n_{s}^{2}(\mathbf{r})\left|\mathbf{E}_{s}(\mathbf{r})\right|^{2} d V\right]\left[\int_{V_{\mathrm{tot}}} n_{p}^{2}(\mathbf{r})\left|\mathbf{E}_{p}(\mathbf{r})\right|^{2} d V\right]}{a n_{s, S i}^{2} n_{p, S i}^{2} \int_{V_{S i}} \mathbf{E}_{s}^{*}(\mathbf{r}) \xi_{R} \vdots \mathbf{E}_{p}(\mathbf{r}) \mathbf{E}_{p}^{*}(\mathbf{r}) \mathbf{E}_{s}(\mathbf{r}) d V},
$$

where $a$ is the lattice period, $n$ is the material refractive index (3.48 for silicon), $\xi_{R}$ is a normalized version of the Raman susceptibility tensor $\chi_{R}{ }^{7} V_{\text {tot }}$ is the volume of one $\mathrm{PhC}$ unit cell, and $V_{\mathrm{Si}}$ is the silicon volume in the unit cell.

This definition of $A_{R}$ can be derived from coupled mode analysis along the guidelines outlined in Ref. 5, and is equivalent to $V_{R} / a$, with $V_{R}$ the Raman volume defined in other works. ${ }^{5,7} A_{\mathrm{TPA}}$ and $A_{\mathrm{FCA}}$ are defined in a similar way to $A_{R},{ }^{7,21}$ and we take into account the polarization dependence of TPA (Ref. 22) with a normalized susceptibility $\xi_{\text {TPA }}$. When substituting the coefficients from Eqs. (8) into (6) and (7), $A_{\text {eff, } p}$ cancels out everywhere, and only the factors $A_{k}$ remain to play the role of effective areas. Note that the factors $A_{k}$ are conceptually very different from the mode area $A_{\text {eff }}$ : the Raman effective area $A_{R}$, in particular, accounts not only for the overlap of the pump and signal modes with each other and with the silicon fraction of the total volume, but also for the selection rules of the Raman effect in silicon through $\xi_{R}$. As a consequence, for the same design and same modes, $A_{R}$ may vary with the waveguide orientation along the silicon plane, as will be discussed later in this section.

We now apply this formalism to estimate the gain achievable in the fundamental TE mode of a silicon $\mathrm{W} 1 \mathrm{PhC}$ waveguide, the dispersion of which can be easily engineered by acting on the position and size of the first two rows of holes adjacent the line defect. ${ }^{23,24}$ In the following, all dispersion curves and complex field amplitudes $\mathbf{E}(\mathbf{r})$ are calculated using the freely available MIT photonics band (MPB) code, ${ }^{25}$ whereas the propagation loss $\alpha(S)$ is estimated with a model based on the code for loss engineering developed in Ref. 14. The code accounts for both out-of-plane radiation losses and backscattering losses and predicts the ensemble average response of a design, without the statistical transmission fluctuations due to multiple backscattering that are particular of each fabricated device. ${ }^{9,26}$ Taking into account both variations in mode profiles and a loss calculation method that well matches experimental data allows us to give a realistic estimate of the achievable Raman gain in true slow-light PhC devices.

Figure 2(a) shows the TE band structure of a standard suspended-membrane W1 waveguide of period $a=412 \mathrm{~nm}$, radius $r=0.286 a$, and slab thickness $220 \mathrm{~nm}$, with its fundamental gap mode lying in the $\sim 1550$-nm wavelength range (middle shaded band). Placing the pump beam onto this fundamental mode and slowing it down does not offer any advantages since there is no obvious guided mode with even symmetry at the Stokes wavelength $(\sim 1680 \mathrm{~nm})$ available that could host the signal ${ }^{27}$ (bottom shaded band). We instead place the signal onto the fundamental TE mode at the $\sim 1550-\mathrm{nm}$
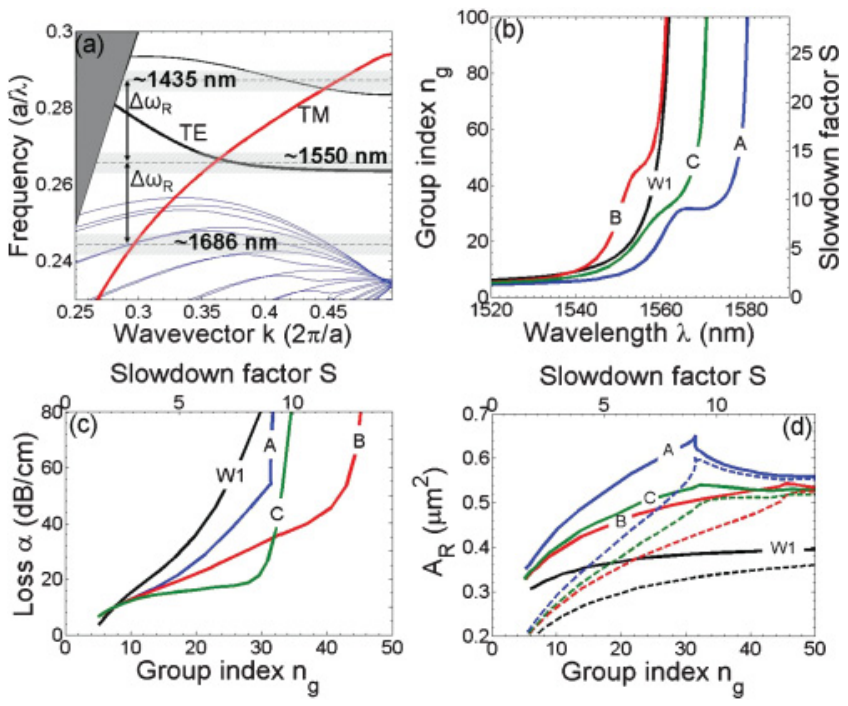

FIG. 2. (Color online) (a) TE band diagram of a membraned W1 waveguide with period $a=412 \mathrm{~nm}$ and hole radius $r=0.286 a$. The fundamental index guided TM mode is also shown in red. The middle shaded band indicates the $\sim 1550$-nm wavelength range; the top and bottom shaded bands are, respectively, blueshifted and redshifted from the middle one by $\Delta \omega_{R}$. (b) Group index and (c) propagation loss of the W1 waveguide and engineered designs A, B, and C. (d) Raman effective areas calculated for waveguides aligned along the standard [110] direction of crystalline silicon (solid line) and along the [100] direction (dashed line). 


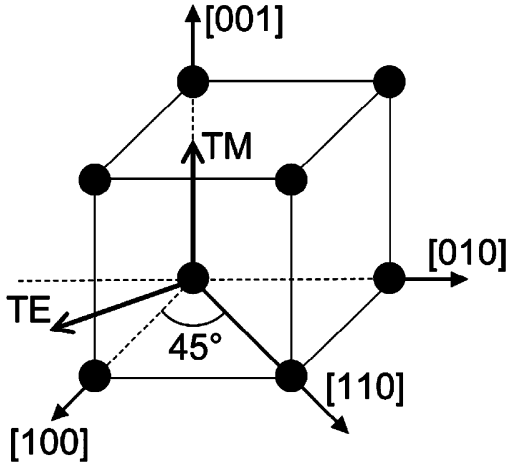

FIG. 3. Crystal axes in a cubic unit cell as used to describe waveguide orientation in the diamond cubic crystal structure of silicon. In practical devices, waveguides are usually aligned along the [110] direction, due to favorable cleaving properties. The corresponding TE and TM mode polarization directions are also indicated. Our analysis also considers aligning the $\mathrm{PhC}$ waveguides along the [100] direction, i.e., $45^{\circ}$ to the cleaving plane.

wavelength range and position the pump in the $\sim 1435$-nm wavelength range (top shaded band) onto the fast fundamental index guided TM mode (red curve). ${ }^{6}$

Figure 2(b) shows the group index of the fundamental TE mode of the same waveguide, together with the group indices for three other waveguides, namely, A, B, and C, which have been engineered to obtain slow light away from the band edge. The corresponding loss curves are shown in Fig. 2(c). The period of designs A, B, and C is reduced to $404 \mathrm{~nm}$ in order for the slow light to be in the same wavelength range as for the $\mathrm{W} 1$. The dispersion of waveguide $\mathrm{A}$ has been designed to exhibit a large region of constant group index around $n_{g}=30$ by choosing $r=0.282 a, s_{1}=-0.12 a$, and $s_{2}=0$, where $s_{1}$ and $s_{2}$ are the lateral shifts of the first and second row of holes, respectively. ${ }^{24}$ Waveguide $\mathrm{B}(r=0.286 a$, $r_{2}=0.26 a, s_{1}=-0.1 a, s_{2}=0.08 a$, with $r_{2}$ the radius of the second row of holes) possibly comes closest to the simplified analysis considered in the preceding section since its loss curve $\alpha_{s}\left(S_{s}\right)$ is very close to linear [Fig. 2(c)], whereas in waveguide $\mathrm{C}$ (same as $\mathrm{B}$, but $r_{2}=0.24 a$ ), the loss $\alpha_{s}$ is almost constant with $S_{s}$. The motivation for the different types of design studied here is to establish the Raman gain that can be achieved with existing realistic waveguides (W1, A) and as to which performance might be possible with more advanced designs (B, C).

Figure 2(d) (solid curves) shows the effective Raman areas calculated for waveguides oriented along the [110] direction of crystalline silicon (Fig. 3), which is the standard practice in real devices due to favorable cleaving properties in this direction. For a fixed design, different group indices are obtained by sweeping the signal wavelength along the curves of Fig. 2(b).

The group index of the TM pump mode has values $n_{g p}$ between 5 and 5.5 and its loss $\alpha_{p}$ is assumed to be $5 \mathrm{~dB} / \mathrm{cm}$, comparable to that of a good fast TE mode. ${ }^{28}$ The TM loss can indeed be considered constant as the TM mode has no significant variation of group index or mode profile in the wavelength range considered. Parameters $g_{R}, \beta_{\mathrm{TPA}}, \sigma_{p, s}$, and $\tau_{c}$ are as defined in Table I.

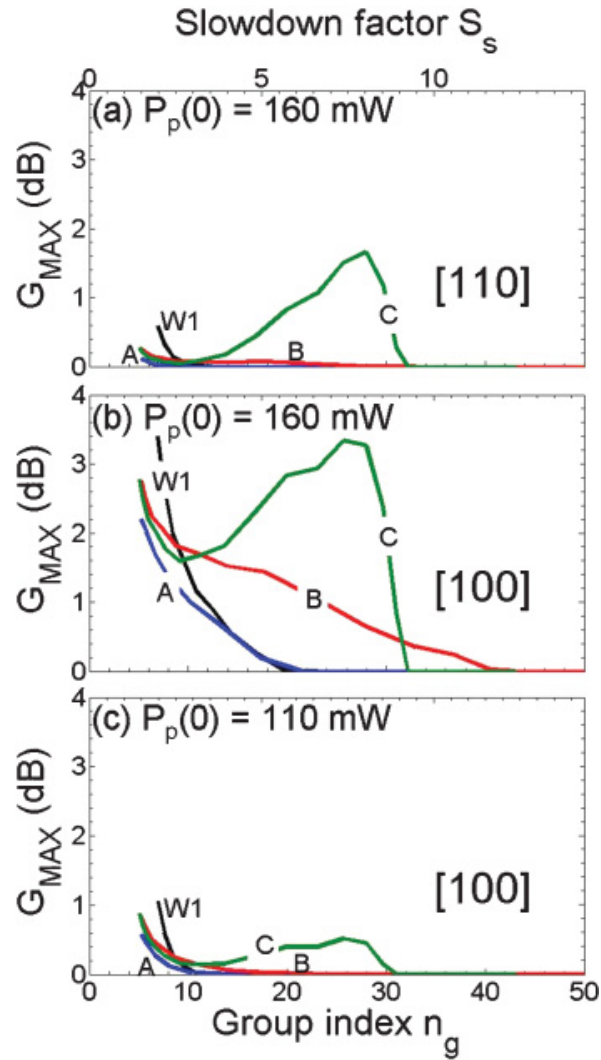

FIG. 4. (Color online) (a) Peak net signal gain for the different designs at input pump power $P_{p}(0)=160 \mathrm{~mW}$. Waveguides are aligned along the standard [110] direction of silicon. The best performance is that of design $\mathrm{C}$, with a gain of $1.66 \mathrm{~dB}$ at length $3.55 \mathrm{~mm}$ in its slow-light regime. (b), (c) Same as (a), but waveguides are aligned along the [100] direction. Pump powers are (b) $160 \mathrm{~mW}$ and (c) $110 \mathrm{~mW}$. At $160 \mathrm{~mW}$ (b), design $\mathrm{C}$ (green) reaches $3.34 \mathrm{~dB}$ gain in the slow-light regime at length $5.04 \mathrm{~mm}$; the fast W1 (black) reaches $3.40 \mathrm{~dB}$ gain at length $6.98 \mathrm{~mm}$.

Solving Eqs. (6) and (7) numerically for the described structures, over a maximum length of $4 \mathrm{~cm}$ and for an input signal power $P_{s}(0)=1 \mathrm{nW}$, leads to the result depicted in Fig. 4(a). Here, we plot the maximum achievable net gain of the signal $G_{\mathrm{MAX}}=10 \log \left[P_{s \mathrm{MAX}} / P_{s}(0)\right]$, with $P_{s \mathrm{MAX}}$ the maximum signal power reached along the waveguide, as a function of signal slowdown factor $S_{s}$ for input pump power $P_{p}(0)=160 \mathrm{~mW}$. The overall performance is now mainly determined by the interplay between the signal propagation loss $\alpha_{s}$ (how much loss must be overcome) and the factor $S_{p} S_{s} / A_{R}$ (which enters the gain term, i.e., how fast the loss can be overcome).

Let us compare the performance of the different designs. The W1 waveguide exhibits the highest loss, but its $A_{R}$ is the lowest; this allows for a small gain to be achieved at moderate $n_{g}$. Waveguide A has lower loss than the W1 over the entire slow region due to reduced backscattering for the same group index ${ }^{14}$ but the penalty of a much higher $A_{R}$ prevents it from showing appreciable net gain, at least in the important low pump-power regime considered here. In waveguide $\mathrm{B}$, the loss curve $\alpha_{s}\left(S_{s}\right)$ is very close to linear [Fig. 2(c)], but its effective Raman area $A_{R}$ also increases with $S_{s}$; hence, the 
small gain $G_{\mathrm{MAX}}$ tends to remain constant within the slow-light region rather than increasing for higher $S_{s}$. Finally, in design $\mathrm{C}$, the increase of $A_{R}$ is counteracted by a loss $\alpha_{s}$ that is almost constant for increasing $S_{s}$, resulting in a clear benefit of slowing down the signal, with a gain $G_{\mathrm{MAX}}=1.66 \mathrm{~dB}$ for $S_{s}=8$ and $\mathrm{CW}$ input pump power $P_{p 0}=160 \mathrm{~mW}$, over a length $L=3.55 \mathrm{~mm}$. Note that waveguide $\mathrm{C}$, for which we estimate the best SRS performance in the slow-light regime, is designed to exhibit lowest loss rather than highest group index.

We have then conducted the same analysis for the same designs but with the waveguides aligned along the [100] crystalline direction of silicon (Fig. 3), i.e., $45^{\circ}$ to the cleaving plane. This additional investigation is motivated by the results obtained in Ref. 7. Here, Checoury et al. show that orienting a W0.66 PhC waveguide along the [100] direction, which in weakly confined waveguides would forbid Raman interaction between a TE pump and a TE Stokes, results in a better Raman TE-TE exchange than in the standard [110] direction due to the presence of a strong longitudinal component in the TE-like mode of a narrow $\mathrm{PhC}$ waveguide.

We extend the study of Checoury et al. by showing that the same concept applies to the TM-pump-TE-signal configuration in the designs considered so far. When the waveguides are aligned along the [100] direction, the Raman effective area of all designs is considerably reduced [Fig. 2(d), dashed curves], resulting in higher peak gains for the same pump power $P_{p}(0)=160 \mathrm{~mW}$ [Fig. 4(b)], and moderate gain appearing already at lower pump power [Fig. 4(c)]. With respect to the case of Ref. 7 , here the difference between the two waveguide orientations is to be attributed mainly to the strong longitudinal component of the TMlike pump mode rather than of the TE-like signal mode (which is nevertheless strong), since Raman selection rules impose that, for a true TM pump (i.e., with no longitudinal components), the scattering efficiency into a TE mode does not depend on the TE polarization direction in the silicon crystal plane. $^{29}$

Note that the reduction in effective Raman area $A_{R}$ is more pronounced for lower group indices [Fig. 2(d)], which results in the performance of the fast $\mathrm{W} 1$ in the [100] direction being comparable to that of the slow engineered design $\mathrm{C}$ [Fig. 4(b)]. At an input CW pump power of $160 \mathrm{~mW}$, we calculate $G_{\mathrm{MAX}}=3.40 \mathrm{~dB}$ at length $6.98 \mathrm{~mm}$ for the fast $\mathrm{W} 1\left(S_{s}=2\right)$ and $G_{\mathrm{MAX}}=3.34 \mathrm{~dB}$ at length $5.04 \mathrm{~mm}$ for design $\mathrm{C}$ in the slow-light regime $\left(S_{s}=7.4\right)$. Once again, this is mainly due to the combination of (very) low propagation loss and low $A_{R}$ (i.e., well-confined mode profile) of the fast $\mathrm{W} 1$ as compared to the slowdown factor $S_{s}$ of design C. It is worth pointing out, however, that within the measurement uncertainty of our experimental data, the propagation loss $\alpha$ of the fast-light regime can be higher than estimated here, resulting in the fast $\mathrm{W} 1$ showing lower gain at these low powers. Still, this does not exclude the existence of a window where fast light in $\mathrm{PhC}$ waveguide may as well be useful for signal amplification through SRS.

When compared to the current record for CW SRS gain, which is $3.7 \mathrm{~dB}$ for $724 \mathrm{~mW}$ pump power into a $4.8-\mathrm{cm}$-long rib waveguide and using a $\mathrm{p}-\mathrm{i}-\mathrm{n}$ diode biased at $-25 \mathrm{~V}$ for carrier lifetime reduction, ${ }^{29}$ our estimate looks very promising indeed; we show that slow-light waveguides can achieve similar gain for lower pump powers AND much shorter device length. Even better, yet higher gain (or identical gain at lower pump thresholds) will be possible if both the pump and the signal can be slowed down simultaneously, thus offering a real prospect for low pump-power operation of silicon Raman amplifiers. Alas, the realization of waveguides that offer the simultaneous slowdown of both pump and Stokes waves is yet challenging.

\section{CONCLUSIONS}

We have provided a simple picture to describe the main difference in the role played by the slowdown factors of Raman pump and Stokes signal in determining the total net gain achievable through SRS in silicon slow-light structures. Slowing down the pump can significantly reduce the pump threshold, allowing for net gain to be achieved at lower powers than would be possible with an equivalent fast waveguide or by slowing down the signal alone. On the other hand, since the maximum gain always increases with signal slowdown factor, which is not necessarily true for the pump, slowing down the signal may result in greater benefit than slowing down the pump for the same input power (above threshold). Ultimately, the real potential for slow-light effects lies in the slowing down of both the pump and the Stokes beams, as the resulting enhancement effects will combine superadditively; this is an exciting but difficult task, however, as it requires dispersion engineering principles to be applied to two different modes, which is a task that has yet to be achieved.

We have then shown that the variations in mode profile with slowdown factor occurring in $\mathrm{PhC}$ waveguides can not be neglected when trying to quantify the benefit of slow light for SRS. By taking into account these mode-profile variations, together with realistic propagation losses, we have performed an estimate of the achievable Raman gain in a real-life PhC slow-light waveguide.

In particular, we have shown that when dispersion engineering is accompanied by loss engineering (design $\mathrm{C}$ ), the detrimental effects of mode-profile variations with slowdown factor can be successfully overcome, making slow light advantageous over fast light at low pump powers. If our prediction holds, this type of design will represent an important step toward achieving Raman gain at low pump powers, thus offering a real prospect for useful application of silicon Raman amplifiers in integrated devices. Naturally, while the emphasis here was on achieving Raman gain for low pump powers, the benefit of slow-light effects will be even more pronounced for higher pump powers.

\section{ACKNOWLEDGMENTS}

The authors would like to thank T. P. White, J. Li, A. Di Falco, and K. Welna for fruitful discussion. This work was in part supported by the UK Engineering and Physical Sciences Research Council (EPSRC) programme UK Silicon Photonics and in part by FWO-Vlaanderen, which provides an Aspirant grant to Y. Lefevre and a postdoctoral Onderzoeker grant to N. Vermeulen. 
*ir21@st-andrews.ac.uk

${ }^{1}$ T. F. Krauss, Nat. Photonics 2, 448 (2008).

${ }^{2}$ J. Li, L. O'Faolain, I. H. Rey, and T. F. Krauss, Opt. Express 19, 4458 (2011).

${ }^{3}$ C. Monat, B. Corcoran, M. Ebnali-Heidari, C. Grillet, B. J. Eggleton, T. P. White, L. O'Faolain, and T. F. Krauss, Opt. Express 17, 2944 (2009).

${ }^{4}$ B. Corcoran, C. Monat, C. Grillet, D. J. Moss, B. J. Eggleton, T. P. White, L. O'Faolain, and T. F. Krauss, Nat. Photonics 3, 206 (2009).

${ }^{5}$ J. F. McMillan, X. Yang, N. C. Panoiu, R. M. Osgood, and C. W. Wong, Opt. Lett. 31, 1235 (2006).

${ }^{6}$ J. F. McMillan, M. Yu, D. L. Kwong, and C. W. Wong, Appl. Phys. Lett. 93, 251105 (2008).

${ }^{7}$ X. Checoury, Z. Han, and P. Boucaud, Phys. Rev. B 82, 041308 (2010).

${ }^{8}$ M. Krause, A. Petrov, and E. Brinkmeyer, in 6th International Conference on Group IV Photonics (GFP) 2009 (IEEE, San Francisco, 2009).

${ }^{9}$ M. Patterson, S. Hughes, S. Schulz, D. M. Beggs, T. P. White, L. O'Faolain, and T. F. Krauss, Phys. Rev. B 80, 195305 (2009).

${ }^{10}$ Note that, in this context, the strong pump assumption boils down to assuming $S_{p} I_{p} \gg S_{s} I_{s}$ rather than $I_{p} \gg I_{s}$.

${ }^{11}$ S. Roy, A. K. Bhadra, and G. P. Agrawal, J. Opt. Soc. Am. B 26, 17 (2009).

${ }^{12}$ S. G. Johnson, M. L. Povinelli, M. Soljai, A. Karalis, S. Jacobs, and J. D. Joannopoulos, Appl. Phys. B: Lasers Opt. 81, 283 (2005).

${ }^{13}$ L. C. Andreani and D. Gerace, Phys. Status Solidi B 244, 3528 (2007).

${ }^{14}$ L. O'Faolain, S. A. Schulz, D. M. Beggs, T. P. White, M. Spasenović, L. Kuipers, F. Morichetti, A. Melloni, S. Mazoyer, J. P. Hugonin, P. Lalanne, and T. F. Krauss, Opt. Express 18, 27627 (2010).
${ }^{15}$ M. Notomi, T. Tanabe, A. Shinya, E. Kuramochi, and H. Taniyama, Adv. Opt. Technol. 2008, 568936 (2008).

${ }^{16}$ Nevertheless, further increase of $S_{p}$ may be advantageous over short lengths [Fig. 1(e)].

${ }^{17}$ R. Claps, D. Dimitropoulos, Y. Han, and B. Jalali, Opt. Express 10, 1305 (2002).

${ }^{18}$ R. Claps, D. Dimitropoulos, V. Raghunathan, Y. Han, and B. Jalali, Opt. Express 11, 1731 (2003).

${ }^{19}$ R. L. Espinola, J. I. Dadap, R. M. Osgood, S. J. McNab, and Y. A. Vlasov, Opt. Express 12, 3713 (2004).

${ }^{20}$ R. M. Osgood, N. C. Panoiu, J. I. Dadap, X. Liu, X. Chen, I. W. Hsieh, E. Dulkeith, W. M. Green, and Y. A. Vlasov, Adv. Opt. Photon. 1, 162 (2009).

${ }^{21} \mathrm{X}$. Yang and C. W. Wong, Opt. Express 15, 4763 (2007).

${ }^{22}$ Q. Lin, O. J. Painter, and G. P. Agrawal, Opt. Express 15, 16604 (2007).

${ }^{23}$ L. H. Frandsen, A. V. Lavrinenko, J. Fage-Pedersen, and P. I. Borel, Opt. Express 14, 9444 (2006).

${ }^{24}$ J. Li, T. P. White, L. O'Faolain, A. Gomez Iglesias, and T. F. Krauss, Opt. Express 16, 6227 (2008).

${ }^{25}$ S. G. Johnson and J. D. Joannopoulos, Opt. Express 8, 173 (2001).

${ }^{26}$ S. Mazoyer, P. Lalanne, J. Rodier, J. Hugonin, M. Spasenović, L. Kuipers, D. Beggs, and T. Krauss, Opt. Express 18, 14654 (2010).

${ }^{27}$ The fundamental TM mode has here too large an effective area to give useful net gains.

${ }^{28}$ L. O'Faolain, X. Yuan, D. McIntyre, S. Thoms, H. Chong, R. M. De La Rue, and T. F. Krauss, Electron. Lett. 42, 1454 (2006).

${ }^{29}$ A. Liu, H. Rong, R. Jones, O. Cohen, D. Hak, and M. Paniccia, J. Lightwave Technol. 24, 1440 (2006). 